\title{
EFFECTIVENESS OF VIDEO AND POSTER EDUCATIONAL PRO- GRAMS IN DENTAL CARIES PREVENTION AMONG PRIMARY SCHOOL STUDENTS
}

\author{
Ossama Ahmmad Muhammad Al-Said, Alaa Eldeen Abdallah, Abd Elhamid Abo Alyazid
}

\begin{abstract}
Objective: To analyze the effectiveness of video and poster performance as educational programs in oral health services in the prevention of dental caries and ensuring good oral hygiene through improving the school children behavior. Materials and Methods: This study was carried out through using two educational materials (video and poster) as two educational methods. Questionnaire was used to evaluate knowledge, attitude, and practice. Microbial samples from saliva were collected to evaluate the reduction in Streptococcus mutans count and Lactobacillus count after the exposure to the video and poster educational materials. Results: The results of the present study revealed an improvement in KAP, as well as, a marked reduction in count of streptococcus mutans and Lactobacillus count after exposure to the tow different educational methods. Conclusion: the use of video and poster as educational materials are effective in reduction of incidence of dental caries.
\end{abstract}

Key Words: Dental cries; Educational programs; Poster, Video

\section{INTRODUCTION}

Dental caries is considered one of the most important oral health problems not only because of their high prevalence among the children, but also because it negatively affects the quality of life as well as high treatment costs ${ }^{(1)}$. The education of healthy behaviors to school children such as; decrease sugar consumption, increase vegetable intake, and daily toothbrushing can easily prevent the incidence of dental cries ${ }^{(2,3)}$.

The implementation of the educational health programs in the educational map in the primary schools could have a strong effect in dental caries prevention among children through increasing their knowledge, change their attitude to healthy behaviors, and motivate their proper practice leading to prevention ${ }^{(1,4)}$. It was found that the educational program approach of oral health could be effective in decreasing the incidence of dental caries through the motivation of the children to change their attitude and improve their practice as a result to increase of their knowledge about the problems of bad oral hygiene as well as unhealthy foods ${ }^{(5)}$.

Recently, oral health education has been considered to be an important and integral part of oral health services that could be delivered to the targeted groups as children in primary schools. The educational interventions used in dental services to change the targeted broad goals such as (KAP) "knowledge, attitude, and practice"; these in pioneering concern with the prevention of oral disease the adoption of healthier life-styles ${ }^{(6)}$.

It was found that the audio-visual aids like; videos and posters, are most effective tools for developing unloosening communication and interaction between student and content as well as student and

\footnotetext{
* Dentist, Egyptian Ministry of Health.

** Assistant Professor, Department of Pedodontics and Oral Health Faculty of dentistry, Al-Azhar University, Cairo, Boy.

*** Lecturer, Department of Pedodontics and Oral Health Faculty of dentistry, Al-Azhar University, Cairo, Boy.
} 
teacher. These aids not only help to increase the children knowledge by the time but also help in developing curiosity to practice the learned lessons, and motivate the children to improve their attitude ${ }^{(7)}$. So, the proper oral hygiene education in primary schools could be an important approach in shifting the level of caries incidence into significantly lower values ${ }^{(8)}$.

Dental education could provide knowledge about the cause of dental caries and the role of unhealthy foods as candy sugars as well as role of bacteria in attacking the targeted tooth ${ }^{(9)}$. In addition, it could explain the role of toothbrushing in caries prevention in young children through elimination of cariogenic organisms and prevention the tooth from their invation ${ }^{(10)}$.

However, it was proved that the proper oral health education alone does not necessarily lead to desirable health behaviors. However, knowledge gained may serve as a tool to empower population groups with accurate information about health and health care strategies, enabling them to act to protect their health ${ }^{(6)}$.

So, the present study was conducted to evaluate the efficacy of oral health education programs in improvement the oral health knowledge, attitude, and practice, as well as its efficacy to induce an effect on school children behavior to decrease caries incidence.

\section{MATERIALS AND METHODS}

The present study was carried out involving 100 children with age ranged from 7 to 10 years old from both sexes. They were student from Governmental school in Suhag, upper-Egypt, "Bardis Elementary school".

\section{Educational Methods:}

The students in this study were divided into two main equal groups according to the educational methods as follows: ${ }^{(9)}$
- Video Group: consisted of 50 students who received "Videos Health Education". two videos named "Soska" and "Senety" were projected on the projecting screen. Then, the children watched the toothbrushing video on the same projecting screen.

- Poster Group: consisted of 50 students which was received "Posters Health Education". A total of 7 printed posters designed and printed by the "Egyptian Society for Pediatric Dentistry and Children with special needs" in collaboration with the FDI-World dental federation and Unilever company, were explained by the researcher while they were placed on the blackboard. The posters have information about the etiology of dental caries, methods of prevention, healthy and unhealthy food, the appropriate time of tooth brushing. Then, the children had direct explanation with images of the proper brushing technique was shown to the children.

\section{Study Design:}

The present study was conducted over two visits with two weeks between the visits and was divided into two main parts:

\section{Questionnaire based on KAP:}

During the first visit; children were given a selfdesigned questionnaire before the exposure to the different education materials. The questionnaire is formed of 12 closed ended, multiple-choice questions categorized into three types, questions targeting Knowledge, questions targeting Attitude and questions targeting Practice. The method to fill the questionnaire by choosing only one answer of the three available was explained to the children. Then, the education materials (posters/ videos) were shown to them in their proper classrooms without mobilizing them. During the second visit; a new copy of the questionnaire was distributed, the method of filling was re-explained. 


\section{Microbiology Sample Collection:}

During the first visit; the microbiology samples of saliva were taken before the exposure to the two different education methods. Then, the education materials (posters/ videos) were shown to them as mention before, and the children received toothbrushes and toothpaste. During the second visit; the collection of saliva samples was done for each group separately to evaluate the efficacy of each health educational interventional program separately.

\section{Microbiological Procedures:}

The sample was transported to the laboratory immediately after collection and processed on same day. The sample was vortexed (15 seconds) and diluted for three different laboratory dilutions (1:1000, 1:10000, and 1:100000) in isotonic saline solution prior to inoculation ${ }^{(11)}$.

\section{A-Streptococcus mutans Count:}

The saliva samples for $S$. mutans count test were diluted for three different laboratory dilutions $(1: 1000,1: 10000$, and 1:100000) using isotonic saline solution. About $0.1 \mathrm{ml}$ of each dilution of the saliva samples was spread homogenously on the surface of appropriate medium (Mitis Salivarius agar with potassium tellurite medium), which is the selective medium to $S$. mutans. Then, the plates were incubated in an anaerobic atmosphere using (BBL gas packs plus, anaerobic system envelope with palladium catalyst) at $37^{\circ} \mathrm{C}$ for 48 hours. After that, $S$. mutans colonies were identified and count and the number of the colony forming units (CFU)/ $\mathrm{ml}$ of the original saliva was calculated using a colony counter.

\section{B-Lactobacillus Count:}

The saliva samples for lactobacillus count test were diluted for three different laboratory dilutions $(1: 1000,1: 10000$, and 1:100000) using isotonic saline solution. About $0.1 \mathrm{ml}$ of each dilution of the saliva samples was spread homogenously on the surface of appropriate medium (Rogosa $S L$ agar medium), which is the selective medium to lactobacillus. Then, the plates were incubated anaerobically at $37^{\circ} \mathrm{C}$ for 48 hours as mention before. After that, lactobacillus colonies were identified and count and the number of the colony forming units $(\mathrm{CFU}) / \mathrm{ml}$ of the original saliva was calculated was using a colony counter.

\section{Statistical Analysis}

Data was recorded and statistically analyzed using IBM SPSS advanced statistics (statically package for social science), version21 (SPSS Inc., Chicago, IL).

\section{RESULTS}

\section{1- KAP Results:}

Through the present study, there were significant improvement in knowledge in both video and poster groups after exposure to the educational materials. However, it was found a statistically significant improvement in knowledge of the poster group when compared with the video group $(p=0.001)$ as shown in Table (1).

TABLE (1) The frequencies of Knowledge of different groups.

\begin{tabular}{|c|c|c|c|c|c|c|}
\hline \multirow{2}{*}{ Variables } & \multicolumn{5}{|c|}{ Knowledge } \\
\cline { 2 - 6 } & \multicolumn{2}{|c|}{ Video } & \multicolumn{2}{c|}{ Posters } & \multirow{2}{*}{ p-value } \\
\cline { 2 - 6 } \multicolumn{2}{|c|}{} & $\mathrm{n}$ & $\%$ & $\mathrm{n}$ & $\%$ & \\
\hline \multirow{2}{*}{ Before } & Yes & 168 & $48 \%$ & 159 & $45.4 \%$ & \multirow{2}{*}{$0.496 \mathrm{~ns}$} \\
\cline { 2 - 7 } & No & 182 & $52 \%$ & 191 & $54.6 \%$ & \\
\hline \multirow{2}{*}{ After } & Yes & 204 & $58.3 \%$ & 264 & $76.3 \%$ & \multirow{2}{*}{$<0.001 *$} \\
\cline { 2 - 6 } & No & 146 & $41.7 \%$ & 82 & $23.7 \%$ & \\
\hline \multicolumn{2}{|c|}{$p$-value } & \multicolumn{2}{|c|}{$0.008 *$} & $<0.001 *$ & \\
\hline
\end{tabular}

*; significant $(p<0.05) n s ;$ non-significant $(p>0.05)$

While, the results of the present study showed a significant improvement in the attitude in both video and poster groups after exposure to the educational 
materials. However, it was found improvement in the attitude results of the poster group when compared with the video group but it is statistically nonsignificant ( $p=0.760)$ as shown in Table (2).

TABLE (2): The frequencies of Attitude of different groups.

\begin{tabular}{|c|c|c|c|c|c|c|}
\hline \multirow{2}{*}{ Variables } & \multicolumn{4}{|c|}{ Attitude } \\
\cline { 2 - 6 } \multicolumn{2}{|c|}{} & \multicolumn{2}{|c|}{ Video } & \multicolumn{2}{c|}{ Posters } & \multirow{2}{*}{-value } \\
\cline { 2 - 6 } & $\mathrm{n}$ & $\%$ & $\mathrm{n}$ & $\%$ & \\
\hline \multirow{3}{*}{ Before } & Yes & 41 & $41 \%$ & 38 & $38 \%$ & \multirow{2}{*}{$0.655 \mathrm{~ns}$} \\
\cline { 2 - 6 } & No & 59 & $59 \%$ & 62 & $62 \%$ & \\
\hline \multirow{2}{*}{ After } & Yes & 68 & $68 \%$ & 70 & $70 \%$ & \multirow{2}{*}{$0.760 \mathrm{~ns}$} \\
\cline { 2 - 6 } & No & 32 & $32 \%$ & 30 & $30 \%$ & \\
\hline \multicolumn{2}{|c|}{$p$-value } & $<0.001 *$ & $<0.001 *$ & \\
\hline
\end{tabular}

*; significant $(p<0.05) n s ;$ non-significant $(p>0.05)$

The results of practice questions revealed a significant improvement in the practice in the both educational groups after exposure to the educational materials. While, it was found a statistically nonsignificant improvement in the practice results of the video group when compared to the poster educational group ( $p=0.103$ ) as shown in Table (3).

TABLE (3): The frequencies of Practice of different groups.

\begin{tabular}{|c|c|c|c|c|c|c|}
\hline \multirow{2}{*}{ Variables } & \multicolumn{4}{|c|}{ Practice } \\
\cline { 2 - 6 } & \multicolumn{2}{|c|}{ Video } & \multicolumn{2}{c|}{ Posters } & \multirow{2}{*}{ p-value } \\
\cline { 2 - 6 } \multicolumn{2}{|c|}{} & $\mathrm{n}$ & $\%$ & $\mathrm{n}$ & $\%$ & \\
\hline \multirow{3}{*}{ Before } & Yes & 63 & $42 \%$ & 65 & $43.3 \%$ & \multirow{2}{*}{$0.816 \mathrm{~ns}$} \\
\cline { 2 - 6 } & No & 87 & $58 \%$ & 85 & $56.7 \%$ & \\
\hline \multirow{2}{*}{ After } & Yes & 111 & $74 \%$ & 98 & $65.3 \%$ & \multirow{2}{*}{$0.103 \mathrm{~ns}$} \\
\cline { 2 - 6 } & No & 39 & $26 \%$ & 52 & $34.7 \%$ & \\
\hline \multicolumn{2}{|c|}{ p-value } & $<0.001 *$ & $<0.001 *$ & \\
\hline
\end{tabular}

*; significant $(p<0.05) n s$; non-significant $(p>0.05)$

\section{2- Microbiology Results:}

\section{A- Streptococcus mutans Results:}

The results of streptococcus mutans among the study revealed a statistically significant decrease in the count of $S$. mutans bacteria for both educational groups after exposure for the both educational materials. While, the video group revealed a statistically non-significantly reduction in S. mutans count when compared to the poster educational group ( $p=0.234$ ) as shown in Table (4).

TABLE (4): The frequencies of Streptococcus mutans of different groups.

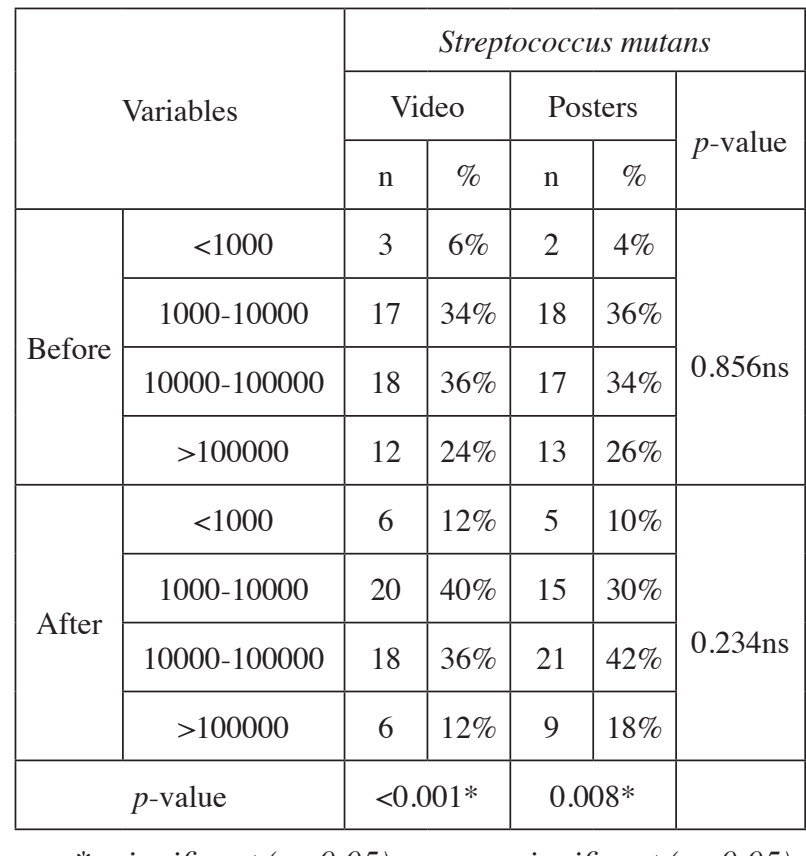

*; significant $(p<0.05) n s ;$ non-significant $(p>0.05)$

\section{B- Lactobacillus Results:}

The results of Lactobacillus among the study revealed a statistically significant decrease in the count of Lactobacillus bacteria for both educational groups after exposure for the both educational materials. While, the video group revealed a statistically significantly reduction in Lactobacillus count when compared to the poster educational group ( $p=0.024)$ as shown in Table (5). 
TABLE (5): The frequencies of Lactobacillus of different groups.

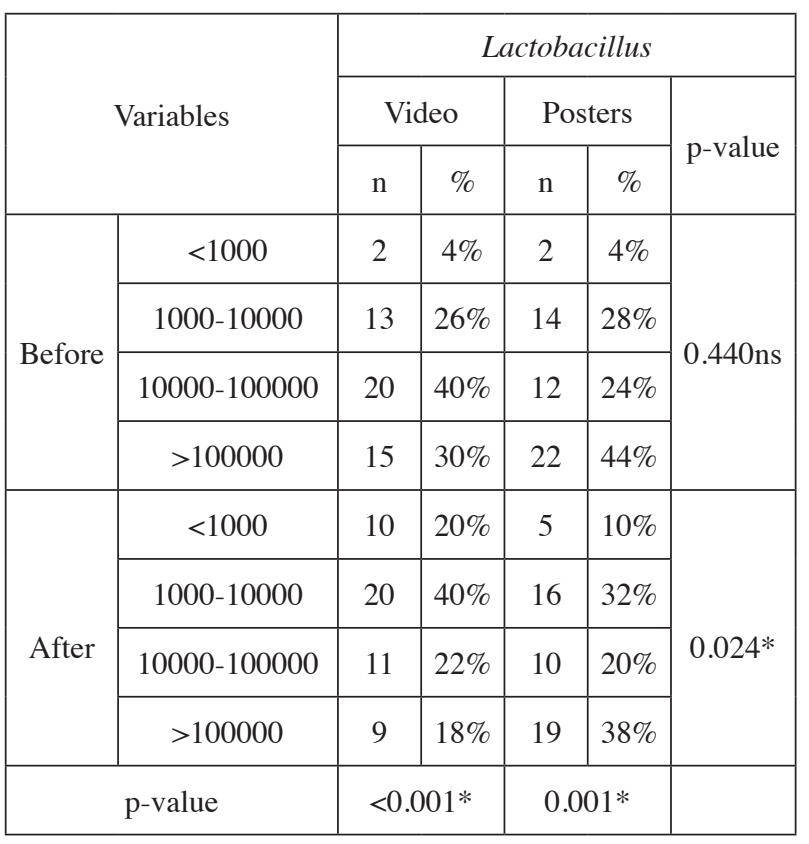

\section{DISCUSSION}

The study of the effectiveness of oral health educational programs as an intervention method helps in the identification of the best educational strategies that could be applied in the oral health practices. Furthermore, the understanding on how this type of intervention should work, in the real world, is crucial to achieve improvements in oral health ${ }^{(1)}$.

Additionally, it was found that, the videos and posters keep the children focused/attentive, and increase the retention rate of the accepted knowledge. Furthermore, they can increase the interaction without fear, so, it improves their attitude. It is also believed that these educational methods carry a high rate of attractiveness that results in a student with increased desire to practice the learned lessons ${ }^{(7)}$.

The findings of the present study suggest that the school is an appropriate structure to implement oral health intervention programs. This in agreement with previous studies which found that; schoolbased intervention has been suggested as a suitable approach for improving children's health and it can be efficient, effective, cost-suitable and beneficial to the entire community ${ }^{(9,12)}$. Furthermore, the previous study in Egypt found that; The oral health education program is an efficient method for improving the oral knowledge, habits and attitude of those children. In addition, Posters illustrations and video cartoon films are effective in communication with those children guided by the assistance of their teacher ${ }^{(9)}$.

In addition, the use of animations and videos during lecture classes would help the students to increase the perception, evoke interest and also break the monotony ${ }^{(13)}$. It is also found that the effectiveness of the lecture depends upon the teacher, regardless of the teaching aid used, and in this context one study points out that what is fundamentally important in university teaching is not the quality of the technology but the quality of the teacher ${ }^{(14)}$.

Questionnaires and indices were used by various authors to assess the outcomes of oral health education (OHE) provided. There are many previous studies which used questionnaires to assess the oral health-related knowledge, attitude, and practice behavior ${ }^{(8)}$.

The results of the present study showed a statistically significant effect of oral health education in student subjected to educational videos as well as educational posters. This in agreement with the results of the previous study in Egypt which revealed that there was a significant positive effect of the oral health education program since more children in both poster and video groups adopted regular oral health behavior such as proper tooth brushing and healthy dietary habits ${ }^{(9)}$. This finding came in agreement with the result of study conducted by Shenoy et al., ${ }^{(15)}$ in India who found that school dental education program was successful in improving oral health knowledge, practices, oral hygiene status, and gingival health of schoolchildren. 
The previous study by Young et al., ${ }^{(16)}$ was found that educational posters can significantly improve the level of knowledge of primary and secondary school teachers in Hong Kong. This agrees with our results of the present study which revealed a statistically significant difference between posters and video groups in knowledge where; the posters group exhibited significantly improve in their knowledge, this may be due to the posters was displayed in the school along the period of study by the teachers (twice in a week), while the videos cannot display.

While, the results of practice in the questionnaire indicate a statistically non-significantly improve in the video group when compare to the poster group. This result may indicate that students are more interesting to watch video, and they tried to repeat tooth brushing according to educational video ${ }^{(14)}$. This in agreement with the results of the previous studies which showed that visual instructions were more effective than written instructions in patients with fixed appliances ${ }^{(14,17)}$.

Improving oral health knowledge in children, even in the short term, is important since research has shown a positive relationship between oral healthrelated knowledge and oral health-related behavior in children ${ }^{(18)}$. Furthermore, healthy behaviors that are developed at a young age are known to be more sustainable in the long term ${ }^{(19)}$.

The importance of, S. mutans, and Lactobacilli and saliva in the development of dental caries has been reviewed extensively through many crosssectional studies ${ }^{(20)}$. The studies have shown that the subjects with active caries tend to harbor higher number of S. mutans and Lactobacilli in their saliva and plaque than those who are caries free. Moreover, longitudinal studies have demonstrated that there is an increase overtime in the number of $S$. mutans and Lactobacilli associated with on- set and progression of caries ${ }^{(21)}$. Numerous studies revealed that the oral health education programs (videos and posters) are an efficient method for improving the oral knowledge, habits and attitude of those children as well as decreasing the caries incidence through decreasing the $S$. mutans and Lactobacillus count $^{(9,22)}$.

In addition, the results of the present study showed a marked reduction in the count of $S$. mutans and Lactobacillus in video educational group, when compared with the poster educational group. This may be due to the fact that the young children are more interested with cartoon videos and they tried to repeat tooth brushing according to educational video $^{(15)}$.

\section{CONCLUSIONS}

1. Oral health education could be effective in prevention of oral diseases in young children.

2. Both video and poster were considered effective education methods.

3. Poster more effective than Video in improving knowledge

4. Video more effective than poster in improving attitude and practice.

5. Schools are potentially perfect places for training and development of healthy habits and behaviors for children at young ages.

\section{REFERENCES}

1. Menegaz AM, Silva AER, Cascaes AM. Educational interventions in health services and oral health: systematic review. RSP. 2018; 52: 52-66.

2. Marinho VC, Higgins JP, Logan S, Sheiham A. Topical fluoride (toothpastes, mouth rinses, gels or varnishes) for preventing dental caries in children and adolescents. Cochrane Database Syst Rev. 2003:4:CD002782.

3. Sheiham A, James WP. A new understanding of the relationship between sugars, dental caries and fluoride use: implications for limits on sugars consumption. Public Health Nutr. 2014; 17:2176-84.

4. Alves GG, Aerts D. As práticas educativas em saúde e a Estratégia Saúde da Família. Cien Saude Colet. 2011;16(1):319-25. 
5. Twetman S. Prevention of early childhood caries (ECC)-review of literature published 1998- 2007. Eur Arch Paediatr Dent. 2008;9(1):12-8.

6. Nakre P, Harikiran A. Effectiveness of oral health education programs: A systematic review. J Int Soc Prev Community Dent. 2013; 3: 103-15.

7. Mohammed R, Rasool I, Meenakshi K, Yasmeen S, Anusha K. Assessment of Student's Perceptions for Audiovisual Aids in Dentistry. Ann Med Health Sci Res. 2017; 7: 256-62.

8. Vanobbergen J, Declerck D, Mwalili S, Martens L. The effectiveness of a 6- year oral health education programme for primary schoolchildren. Comm Dent Oral Epidemiol. 2004; 32:173-82.

9. Farag S, Rashed M, Fouad W. Oral health status of preschool children in El-Suez governorate in relation to dental care given and the influence of oral heath educational program. EDJ. 2014; 60:1103-13.

10. Habibian M, Beighton D, Stevenson R, Lawson M, Roberts G. Relationship between dietary behaviors, oral hygiene, and mutans streptococci in dental plaque of a group of infants in southern England. Arch Oral Biol. 2002; 47:491-98.

11. Sakeenabi B, Hiremath SS. Dental caries experience and salivary streptococcus mutans, lactobacilli scores, salivary ow rate and salivary buffreing capacity among 6-year-old Indian school children. J Clin Exp Dent. 2011;3: e412-7.

12. Moses J, Rangeeth B N and Gurunathan D. Prevalence of dental caries, socio-economic old school going children of chidambaram status and treatment needs among 5 to 15-year-old school going children of Chidambaram. J Clin Diagn Rese. 2011; 5: 146-51.

13. Souza A, Ankolekar V, Kotian S, Souza A, Hosapatna M. Effectiveness of Audio-Visual Aids in Medical Education: A Students'. Inter J Oral Health Res. 2014; 4: 228-34.
14. Pouradeli S, Hashemipour MA, Zolala F, Baneshi MR, Ahmadi- Tabatabaei SV, Habib-Agahi R. Short-term effect of two education methods on oral health among hearing impairment children. J Oral Health Oral Epidemiol. 2016; 5: 221-17.

15. Shenoy RP, Krishnan V, Sequeira PS. Effectiveness of a school dental education program in improving oral health knowledge and oral hygiene practices and status of school children. Indian J Dent Res 2010; 21:253-9.

16. Young C, Wong K, Cheung L. Effectiveness of educational poster on knowledge of emergency management of dental trauma--part 2: cluster randomized controlled trial for secondary school students. PLoS One. 2014;9: e101972-76.

17. Lees A, Rock WP. A comparison between written, verbal, and videotape oral hygiene instruction for patients with fixed appliances. J Orthod 2000; 27: 323-8.

18. Poutanen R, Lahti S, Tolvanen M, Hausen H. Parental influence on children's oral health-related behavior. Acta Odontolologica Scandinavia. 2006; 64: 286-92.

19. Kwan S, Petersen P, Pine C, Borutta A. Health- promoting schools: an opportunity for oral health promotion. Bull World Health Organ. 2005; 83:677-85.

20. Hegde P, Ashok Kumar B, Ankola V. Dental caries experience and salivary levels of streptococcus mutans and lactobacilli in 13-15 years old children of Belgaum city, Karrnataka. J Indian Soc Pedod Prev Dent. 2005; 23:23-6.

21. Gamboa F, Estupinan M, Chaves M, Galindo A. Bacteriocins in S. mutans strains isolated from children with and without dental caries: biotypes and sensitivity to antibiotics. Acta Odontol Latinoam.2008;21:97-104.

22. Bedwani N, Mesenge C, Denis M, Desfontaine J. A pilot educational intervention for dental caries prevention among school children in Alexandria (Egypt). E D J. 2008; 1449:1454-54. 